\title{
Inter- and intramolecular Diels-Alder/retro-Diels-Alder reactions of 4-silylated oxazoles
}

\author{
Pascal C. Ducept ${ }^{\mathrm{a}}$ and Stephen P. Marsden*,a,b \\ a) Department of Chemistry, Imperial College of Science, Technology and Medicine, \\ London SW7 2AY, U. K. \\ b) Current address: Department of Chemistry, University of Leeds, Leeds LS2 9JT, U. K. \\ E-mail: S.P.Marsden@chem.leeds.ac.uk
}

\section{Dedicated to Professor Charles Rees F.R.S. on the occasion of his $75^{\text {th }}$ birthday}

(received 31 May 02; accepted 04 Jul 02; published on the web 12 Jul 02)

\begin{abstract}
4-Silylated oxazoles have been shown to undergo inter- and intramolecular Diels-Alder/retroDiels-Alder reactions with electron-poor alkynes to generate polysubstituted furans. The ease of synthesis of the requisite oxazoles by the rhodium-catalysed condensation of nitriles with silylated diazoacetate greatly increases the scope of this reaction.
\end{abstract}

Keywords: Silylated oxazoles, diazoacetate, furans, Diels-Alder, retro-Diels-Alder

\section{Introduction}

Oxazoles are well recognised for their ability to act as azadienes in Diels-Alder cycloaddition reactions with both alkenes and alkynes. ${ }^{1}$ The adducts from the former class of reactions usually eliminate water to generate substituted pyridines, while the latter class gives substituted furans by retro-Diels-Alder elimination of nitriles. This has proven to be a powerful method for the synthesis of this important class of heterocycles and has been widely used in the context of complex natural product synthesis. ${ }^{2}$

One of the most convenient methods for the synthesis of oxazoles involves the condensation of diazocarbonyl compounds with nitriles, discovered by Huisgen in $1961 .^{3}$ Although the reaction can be carried out under a range of conditions (thermal, photochemical, Lewis-acid catalysed) the use of rhodium (II) carboxylate catalysts pioneered by Helquist ${ }^{4}$ and Moody ${ }^{5}$ offers particularly mild conditions which are compatible with highly functionalised substrates. One limitation of this method is that in general these reactions work best with doubly stabilised diazocarbonyl compounds such as diazomalonates, diazoketoesters and diazoketophosphonates so as to avoid competing carbene dimerisation. ${ }^{6}$ This in turn limits the utility of this method as an 
approach to cycloaddition precursors, since the presence of electron-withdrawing groups on the oxazole deactivates the system toward cycloaddition and also oxazoles bearing carbonyl functions at the 4-position are susceptible to Cornforth rearrangement on thermolysis. ${ }^{7}$ Indeed, to our knowledge there are no known successful examples of cycloadditions of oxazoles bearing a carbonyl group at the 4-position with alkynes, and only two reports of reactions with alkenes. ${ }^{8}$

We have recently shown that 4-silylated oxazoles can be readily prepared by the condensation of silyl diazoacetates with nitriles under rhodium catalysis. ${ }^{9}$ The reluctance of silyl diazoacetates and their derived rhodium carbenoids to undergo dimerisation means that these reactions are operationally simple, requiring no precautions such as high dilution or slow addition of substrate. It therefore became apparent that were these substrates to undergo DielsAlder reactions with alkynes, followed by subsequent retro-Diels-Alder elimination of silyl cyanide, then this would considerably broaden the scope of the overall furan synthesis. We report herein the successful Diels-Alder/retro-Diels-Alder reactions of silylated oxazoles with electronpoor alkynes in both inter- and intramolecular manifolds.

\section{Results and Discussion}

We elected first to study the intermolecular variant. The silylated oxazoles 1a-g were prepared from the corresponding nitrile and ethyl (triethylsilyl)diazoacetate under rhodium (II) octanoate catalysis, according to our standard procedure. ${ }^{9}$ Oxazoles 1a-f were then thermolysed in turn with dimethyl acetylenedicarboxylate 2a under the conditions shown (Scheme 1 and Table 1). Pleasingly, the desired Diels-Alder/retro-Diels-Alder sequence to yield the substituted furans $\mathbf{3}$ was observed in all but two cases. Where successful, the yields of the adducts were moderate to good except in the case of the simple methyl-substituted oxazole $\mathbf{1 b}$, which gave a very messy reaction from which only $18 \%$ of the clean furan could be isolated. Attempted reaction of the corresponding ethyl homologue also gave a messy reaction from which it was not possible to obtain completely pure furan, and it therefore appears that 2-alkyloxazoles are poor substrates for this reaction. As expected, the aryl- and heteroaryl-substituted oxazoles 1a,c required higher temperatures to drive the reactions as a consequence of the loss of stabilising conjugation through the cycloaddition step.

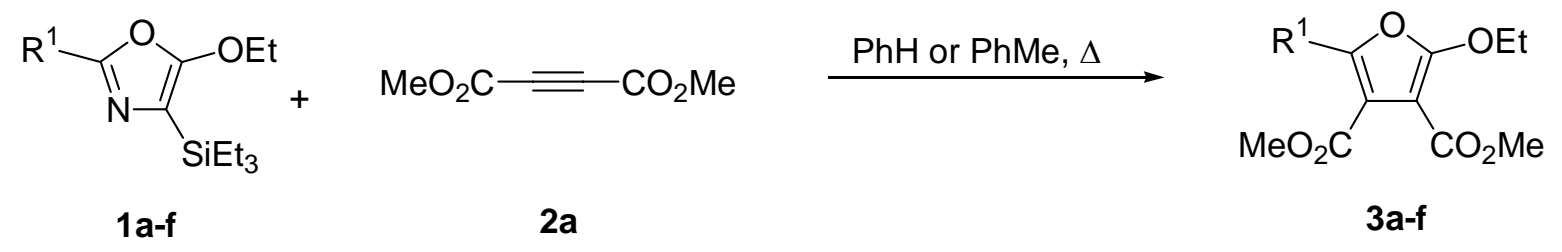

\section{Scheme 1}


Table 1. Cycloaddition of oxazoles 1a-f with dimethyl acetylenedicarboxylate 2a

\begin{tabular}{ccccc}
\hline Entry & $\mathrm{R}^{1}$ & Temp. & Solvent & Yield \\
\hline $\mathrm{a}$ & $\mathrm{Ph}$ & 100 & $\mathrm{PhMe}$ & 65 \\
$\mathrm{~b}$ & $\mathrm{Me}$ & 60 & $\mathrm{PhH}$ & 18 \\
$\mathrm{c}$ & 2-thiophenyl & 120 & $\mathrm{PhMe}$ & 52 \\
$\mathrm{~d}$ & $\mathrm{CO}_{2} \mathrm{Me}$ & 120 & $\mathrm{PhMe}$ & 62 \\
e & 2-furanyl & 110 & $\mathrm{PhMe}$ & $0^{\mathrm{a}}$ \\
$\mathrm{f}$ & $\mathrm{NMe}_{2}$ & 60 & $\mathrm{PhH}$ & 0 \\
\hline
\end{tabular}

${ }^{\text {a }} 43 \%$ yield of 4 isolated (see Scheme 2 ).

The reaction of 2-furanyl oxazole 1e with 2a gave an unoptimised $43 \%$ yield of a 1:1 cycloadduct 4 from addition across the more electron-rich furan ring rather than the oxazole (Scheme 2). The highly electron-rich dimethylamino-substituted oxazole 1f underwent a rapid reaction to produce a new, more polar product as judged by TLC analysis, but despite significant effort this material could not be isolated following column chromatography.

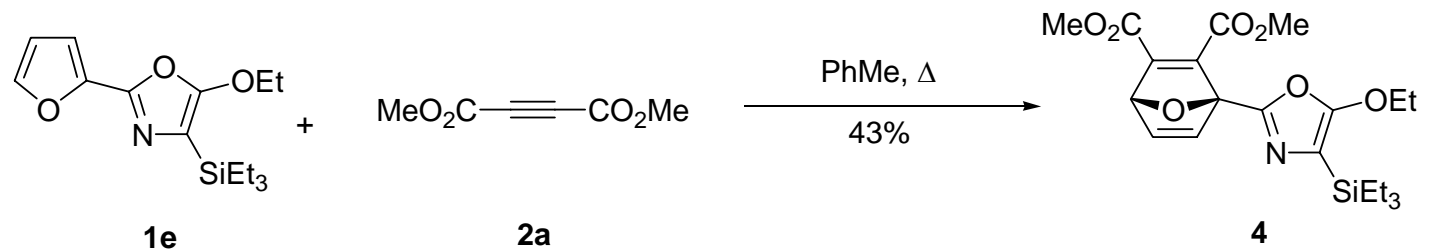

\section{Scheme 2}

We next investigated the intermolecular reactions of oxazoles $\mathbf{1 a} / \mathbf{g}$ with less activated dienophiles. Both oxazoles reacted in a completely regioselective manner with methyl propiolate $\mathbf{2 b}$ to generate the furans $5 \mathbf{a} / \mathbf{g}$ in moderate yield (Scheme 3 and Table 2). The regioselectivity mirrors that previously observed ${ }^{80,10}$ and as expected from the alignment of the electron-rich 2-position of the oxazole with the electron-deficient terminus of the alkyne. Notably, the presence of only a single activating group meant that higher temperatures were required than for the corresponding reactions with 2a. In the light of this, it was felt that less-active dienophiles would be less likely still to undergo cycloaddition and indeed the oxazoles were recovered unchanged from attempted reaction with methyl 3-phenylpropiolate $\mathbf{2 c}$, diphenylacetylene $\mathbf{2 d}$ and trimethylsilylacetylene $\mathbf{2 e .}$<smiles>[R]c1nc([SiH2])c([OH2+])o1</smiles>

$1 \mathrm{a}, \mathrm{g}$<smiles>[R]C#C[R]</smiles>

2b-e
PhMe, PhEt or DCB $\triangle$

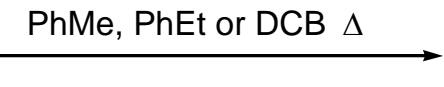

\section{Scheme 3}<smiles>[R]c1oc(OCC)c([R])c1[R]</smiles>

$5 a, b$ 
Table 2. Attempted cycloaddition of oxazoles 1a,g with dienophiles $\mathbf{2 b - e}$

\begin{tabular}{ccccccc}
\hline Entry & $\mathrm{R}^{1}$ & $\mathrm{R}^{2}$ & $\mathrm{R}^{3}$ & Temp. & Solvent & Yield \\
\hline $\mathrm{a}$ & $\mathrm{Ph}$ & $\mathrm{H}$ & $\mathrm{CO}_{2} \mathrm{Me}$ & 160 & $\mathrm{PhEt}$ & 43 \\
$\mathrm{~b}$ & $\mathrm{Et}$ & $\mathrm{H}$ & $\mathrm{CO}_{2} \mathrm{Me}$ & 110 & $\mathrm{PhMe}$ & 40 \\
c & $\mathrm{Ph}$ & $\mathrm{Ph}$ & $\mathrm{CO}_{2} \mathrm{Me}$ & 160 & $\mathrm{PhEt}$ & 0 \\
$\mathrm{~d}$ & $\mathrm{Et}$ & $\mathrm{Ph}$ & $\mathrm{CO}_{2} \mathrm{Me}$ & 160 & $\mathrm{PhEt}$ & 0 \\
$\mathrm{e}$ & $\mathrm{Et}$ & $\mathrm{Ph}$ & $\mathrm{Ph}$ & 180 & $\mathrm{DCB}$ & 0 \\
$\mathrm{f}$ & $\mathrm{Et}$ & $\mathrm{H}$ & $\mathrm{SiMe}_{3}$ & 180 & $\mathrm{DCB}$ & 0 \\
\hline
\end{tabular}

Finally, we turned our attention to intramolecular variants of the reaction. Three potential substrates were prepared, with varying degrees of activation in the dienophile part of the molecule. The diactivated and monoactivated oxazoles $\mathbf{6 a} / \mathbf{b}$ were prepared by the route shown in Scheme 4. Thus, condensation of 4-(tert-butyldimethylsilyloxy)pentanenitrile ${ }^{11}$ with ethyl (triethylsilyl)diazoacetate under rhodium catalysis gave a $79 \%$ yield of the oxazole 7 . Deprotection with TBAF yielded alcohol 8 which was oxidised under Swern conditions to yield aldehyde 9. Addition of lithiated methyl propiolate ${ }^{12}$ or trimethylsilylacetylene to $\mathbf{9}$ gave the alcohols $10 \mathbf{a} / \mathbf{b}$, which were oxidised with Dess-Martin periodinane to give $\mathbf{6 a} / \mathbf{b}$ in good yield.

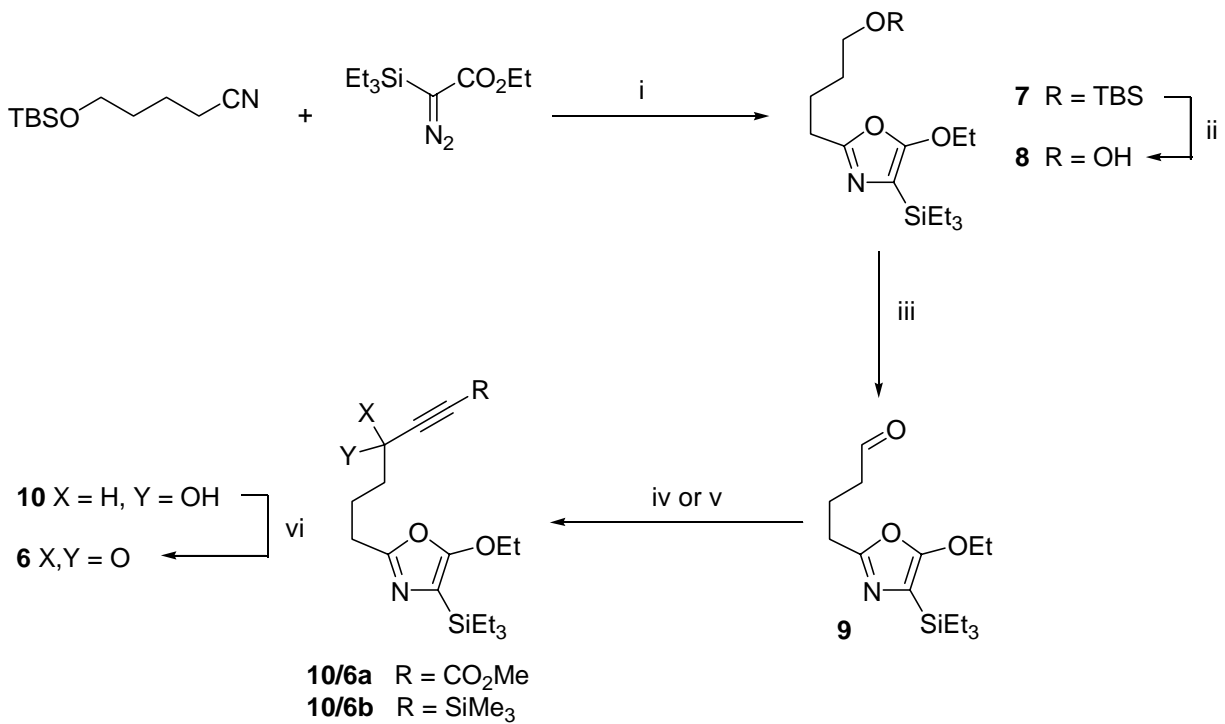

Scheme 4. (i). $1 \% \mathrm{Rh}_{2}$ (oct) $)_{4}$, benzene, 79\%; (ii). TBAF, THF, r.t., 91\%; (iii). $(\mathrm{COCl})_{2}$, DMSO, $\mathrm{CH}_{2} \mathrm{Cl}_{2},-78^{\circ} \mathrm{C}$, then $\mathrm{Et}_{3} \mathrm{~N}$, $-78{ }^{\circ} \mathrm{C}$ to r.t., $73 \%$; (iv). methyl propiolate, ${ }^{\mathrm{n}} \mathrm{BuLi}$, THF/Et ${ }_{2} \mathrm{O} /$ pentane, $-120^{\circ} \mathrm{C}$, then add $9,-120^{\circ} \mathrm{C}$ to $-78{ }^{\circ} \mathrm{C}, 78 \%$; (v). trimethylsilylacetylene, ${ }^{n} \mathrm{BuLi}, \mathrm{THF},-78^{\circ} \mathrm{C}$, then add 9, 90\%; (vi). Dess-Martin periodinane, $\mathrm{CH}_{2} \mathrm{Cl}_{2}, 77 \%(6 \mathbf{6})$ and $80 \%(\mathbf{6 b})$.

The non-activated alkyne $\mathbf{6 c}$ was simply prepared by condensation of 7-phenylhept-6-ynylnitrile $\mathbf{1 1}$ with ethyl (triethylsilyl)diazoacetate to give $\mathbf{6 c}$ in 62\% yield (Scheme 5). 

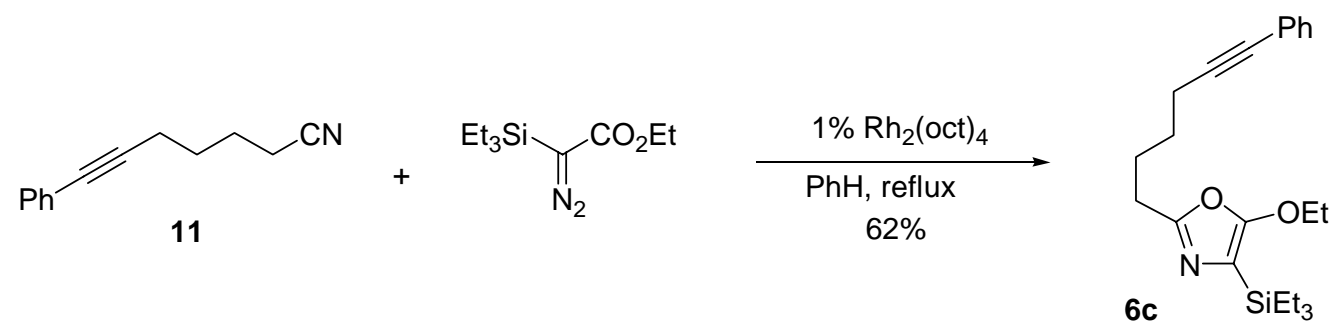

\section{Scheme 5}

The Diels-Alder/retro-Diels-Alder reactions of $\mathbf{6 a} / \mathbf{b}$ proceeded smoothly to give the desired dihydrobenzofuranones 12a/b in good yield (Scheme 6). Notably, in the intramolecular manifold it appears that one activating group (as in $\mathbf{6 b}$ ) is sufficient for effective reaction, though as expected the monoactivated system required higher temperatures (refluxing dichlorobenzene compared to toluene) to drive the reaction. It should also be noted that the silyl function in $\mathbf{1 2 b}$ may potentially be utilised as a handle for the introduction of further functionality through ipsoelectrophilic substitution reactions. ${ }^{9}$ Finally, thermolysis of $\mathbf{6 c}$ failed to give any cycloadduct even under forcing conditions $\left(180^{\circ} \mathrm{C}\right.$ in dichlorobenzene), with only starting material being recovered. This suggests that at least one activating group is required, although further experiments will be required to separate this effect from the entropic factors associated with exchange of the $\mathrm{sp}^{2}$ carbonyl unit for a methylene unit in the linking chain.

\section{Scheme 6}
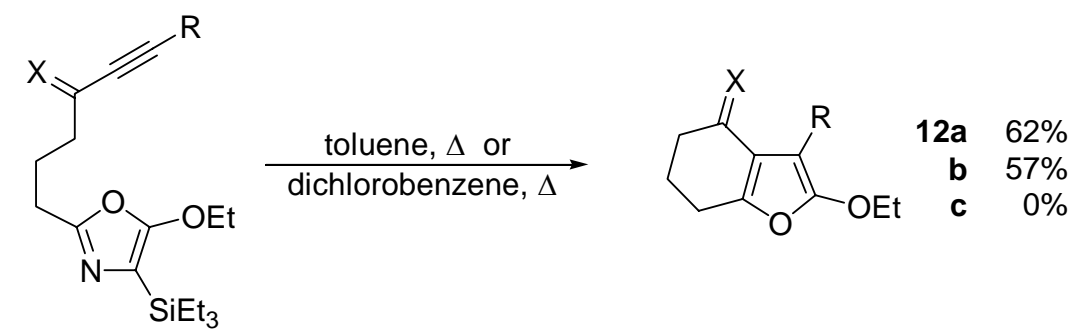

In summary, we have shown that readily available 4-silyl oxazoles will participate in DielsAlder/retro-Diels-Alder sequences, giving facile access to a range of polysubstituted furans in just two steps from nitriles.

\section{Experimental Section}

General procedure for the cycloaddition reactions: 2-ethoxy-3,4-di(methoxycarbonyl)-5phenylfuran (3a)

A solution of 5-ethoxy-2-phenyl-4-triethylsilyloxazole $(0.121 \mathrm{~g}, 0.4 \mathrm{mmol})$ and dimethyl acetylenedicarboxylate $(59 \mu \mathrm{l}, 0.48 \mathrm{mmol})$ in toluene $(0.5 \mathrm{ml})$ was heated to $100^{\circ} \mathrm{C}$. The solution was cooled to room temperature, concentrated under reduced pressure and the residual oil 
purified by flash chromatography (60:40 petroleum ether:diethyl ether) to yield 2-ethoxy-3,4di(methoxycarbonyl)-5-phenylfuran 3a as a white solid (0.079 g, 65 \%). m.p. $74-75^{\circ} \mathrm{C} ; v_{\max }$ (KBr/film) 2987 w, 2952 w, 2904 w, 1724 s (C=O), 1602 s, 1573 w, 1492 w, 1459 m, 1446 m, 1417 w, 1390 w, 1346 m, $1222 \mathrm{~m}, 1097$ s, $1051 \mathrm{~m}, 811 \mathrm{w}, 781 \mathrm{w}, 763 \mathrm{w}, 692 \mathrm{w} \mathrm{cm}^{-1}$; $\delta_{\mathrm{H}}(300$ $\left.\mathrm{MHz}, \mathrm{CDCl}_{3}\right) 1.48\left(3 \mathrm{H}, \mathrm{t}, J=7 \mathrm{~Hz}, \mathrm{OCH}_{2} \mathrm{CH}_{3}\right), 3.78\left(3 \mathrm{H}, \mathrm{s}, \mathrm{CO}_{2} \mathrm{CH}_{3}\right), 3.88\left(3 \mathrm{H}, \mathrm{s}, \mathrm{CO}_{2} \mathrm{CH}_{3}\right)$, $4.51\left(2 \mathrm{H}, \mathrm{q}, J=7 \mathrm{~Hz}, \mathrm{OCH}_{2} \mathrm{CH}_{3}\right), 7.30-7.38\left(3 \mathrm{H}, \mathrm{m}, \mathrm{H}_{3^{\prime}, 4^{\prime}}\right), 7.55\left(2 \mathrm{H}, \mathrm{m}, \mathrm{H}_{2}\right)$ ); $\delta_{\mathrm{C}}(75 \mathrm{MHz}$, $\left.\mathrm{CDCl}_{3}\right) 14.9 \mathrm{OCH}_{2} \underline{\mathrm{CH}}_{3}, 51.5 \mathrm{CO}_{2} \underline{\mathrm{CH}}_{3}, 52.6 \mathrm{CO}_{2} \underline{\mathrm{CH}}_{3}, 68.5 \mathrm{OCH}_{2} \mathrm{CH}_{3}, 93.1 \mathrm{C}_{3}, 114.7 \mathrm{C}_{4}, 125.2$ $\mathrm{C}_{3}, 128.4 \mathrm{C}_{4}, 128.5 \mathrm{C}_{1}^{\prime}, 128.6 \mathrm{C}_{2}{ }^{\prime}, 141.8 \mathrm{C}_{5}, 160.5 \mathrm{C}_{2}, 162.3 \mathrm{CO}_{2} \mathrm{CH}_{3}, 165.1 \underline{\mathrm{CO}}_{2} \mathrm{CH}_{3} ; \mathrm{m} / \mathrm{z}$ $\left(\mathrm{CI}+, \mathrm{NH}_{3}\right) 305\left([\mathrm{M}+\mathrm{H}]^{+}, 100\right), 105(12)$; HRMS (CI+) Found $[\mathrm{M}+\mathrm{H}]^{+}$305.1034; $\mathrm{C}_{16} \mathrm{H}_{17} \mathrm{O}_{6}$ requires 305.1025.

2-Ethoxy-3,4-di(methoxycarbonyl)-5-methylfuran (3b). A solution of 5-ethoxy-2-methyl-4triethylsilyloxazole $(0.097 \mathrm{~g}, 0.4 \mathrm{mmol})$ and dimethyl acetylenedicarboxylate $(51 \mu \mathrm{l}, 0.42 \mathrm{mmol})$ in benzene $(0.2 \mathrm{ml})$ was heated to $60^{\circ} \mathrm{C}$. The crude product was purified by flash chromatography (70:30 petroleum ether:diethyl ether) to yield 2-ethoxy-3,4di(methoxycarbonyl)-5-methylfuran $3 \mathbf{b}$ as a colourless liquid $(0.017 \mathrm{~g}, 18 \%)$. $v_{\max }(\mathrm{KBr} / \mathrm{film})$ 2955 s, 2921 s, 2881 s, 1722 s (C=O), 1611 s, 1512 w, 1447 s, 1331 s, 1216 s, 1088 s, 1024 s, $844 \mathrm{~m}, 811 \mathrm{~m}, 781 \mathrm{~m}, 746 \mathrm{~m} \mathrm{~cm}^{-1}$; $\delta_{\mathrm{H}}\left(270 \mathrm{MHz}, \mathrm{CDCl}_{3}\right) 1.40\left(3 \mathrm{H}, \mathrm{t}, J=7 \mathrm{~Hz}, \mathrm{OCH}_{2} \mathrm{CH}_{3}\right)$, $2.36\left(3 \mathrm{H}, \mathrm{s}, \mathrm{CH}_{3}\right), 3.77\left(3 \mathrm{H}, \mathrm{s}, \mathrm{CO}_{2} \mathrm{CH}_{3}\right), 3.81\left(3 \mathrm{H}, \mathrm{s}, \mathrm{CO}_{2} \mathrm{CH}_{3}\right), 4.35(2 \mathrm{H}, \mathrm{q}, J=7 \mathrm{~Hz}$, $\left.\mathrm{OCH}_{2} \mathrm{CH}_{3}\right) ; \delta_{\mathrm{C}}\left(75 \mathrm{MHz}, \mathrm{CDCl}_{3}\right) 12.8 \mathrm{CH}_{3}, 14.1 \mathrm{OCH}_{2} \mathrm{CH}_{3}, 51.6 \mathrm{CO}_{2} \underline{\mathrm{CH}}_{3}, 51.8 \mathrm{CO}_{2} \mathrm{CH}_{3}, 68.8$ $\mathrm{OCH}_{2} \mathrm{CH}_{3}, 92.4 \mathrm{C}_{3}, 113.8 \mathrm{C}_{4}, 147.5 \mathrm{C}_{5}, 159.7 \underline{\mathrm{CO}}_{2} \mathrm{CH}_{3}, 162.8 \underline{\mathrm{CO}}_{2} \mathrm{CH}_{3}, 163.9 \mathrm{C}_{2} ; \mathrm{m} / \mathrm{z}(\mathrm{CI}+$, $\left.\mathrm{NH}_{3}\right) 243\left([\mathrm{M}+\mathrm{H}]^{+}, 100\right)$; HRMS (CI+) Found $[\mathrm{M}+\mathrm{H}]^{+}$243.0864; $\mathrm{C}_{11} \mathrm{H}_{15} \mathrm{O}_{6}$ requires 243.0869.

2-Ethoxy-3,4-di(methoxycarbonyl)-5-(2-thiophenyl)furan (3c). 5-Ethoxy-2-(2-thiophenyl)-4triethylsilyloxazole $(0.115 \mathrm{~g}, 0.37 \mathrm{mmol})$ and dimethyl acetylenedicarboxylate $(50 \mu \mathrm{l}, 0.4 \mathrm{mmol})$ in toluene $(0.4 \mathrm{ml})$ was heated to $120^{\circ} \mathrm{C}$. The crude product was purified by flash chromatography (50:50 petroleum ether:diethyl ether) to yield 2-ethoxy-3,4di(methoxycarbonyl)-5-(2-thiophenyl)furan 3c as a colourless oil $(0.060$ g, $52 \%)$. $v_{\max }$ (KBr/film) 2989 w, 2952 w, 1722 s (C=O), 1604 s, 1446 s, 1328 s, 1240 s, 1093 s, 707 w cm'; $\delta_{\mathrm{H}}\left(300 \mathrm{MHz}, \mathrm{CDCl}_{3}\right) 1.46\left(3 \mathrm{H}, \mathrm{t}, J=7 \mathrm{~Hz}, \mathrm{OCH}_{2} \mathrm{CH}_{3}\right), 3.78\left(3 \mathrm{H}, \mathrm{s}, \mathrm{CO}_{2} \mathrm{CH}_{3}\right), 3.86(3 \mathrm{H}, \mathrm{s}$, $\left.\mathrm{CO}_{2} \mathrm{CH}_{3}\right), 4.48\left(2 \mathrm{H}, \mathrm{q}, J=7 \mathrm{~Hz}, \mathrm{OCH}_{2} \mathrm{CH}_{3}\right), 7.02\left(1 \mathrm{H}, \mathrm{dd}, J_{4^{\prime}-3^{\prime}}=5 \mathrm{~Hz}, J_{4^{\prime}-5}{ }^{\prime}=3.5 \mathrm{~Hz}, \mathrm{H}_{4^{\prime}}\right)$, $7.30\left(1 \mathrm{H}, \mathrm{dd}, J_{3^{\prime}-4^{\prime}}=5 \mathrm{~Hz}, J_{3^{\prime}-5^{\prime}}=1 \mathrm{~Hz}, \mathrm{H}_{3^{\prime}}\right), 7.43\left(1 \mathrm{H}, \mathrm{dd}, J_{5^{\prime}-4^{\prime}}=3.5 \mathrm{~Hz}, J_{5^{\prime}-3^{\prime}}=1 \mathrm{~Hz}, \mathrm{H}_{5^{\prime}}\right) ; \delta_{\mathrm{C}}$ (75 MHz, $\mathrm{CDCl}_{3}$ ) $14.9 \mathrm{OCH}_{2} \underline{\mathrm{CH}}_{3}, 51.6 \mathrm{CO}_{2} \underline{\mathrm{CH}}_{3}, 52.3 \mathrm{CO}_{2} \underline{\mathrm{CH}}_{3}, 68.9 \mathrm{OCH}_{2} \mathrm{CH}_{3}, 93.3 \mathrm{C}_{3}, 113.2$

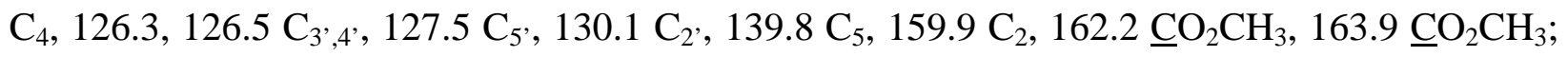
$\mathrm{m} / \mathrm{z}\left(\mathrm{CI}+, \mathrm{NH}_{3}\right) 311([\mathrm{M}+\mathrm{H}]+, 100), 111$ (27); HRMS (CI+) Found $[\mathrm{M}+\mathrm{H}]^{+}$311.0583; $\mathrm{C}_{14} \mathrm{H}_{15} \mathrm{O}_{6} \mathrm{~S}$ requires 311.0589.

2-Ethoxy-3,4,5-tri(methoxycarbonyl)furan (3d). 5-Ethoxy-2-methoxycarbonyl-4-triethylsilyloxazole $(0.144 \mathrm{~g}, 0.5 \mathrm{mmol})$ and dimethyl acetylenedicarboxylate $(73 \mu \mathrm{l}, 0.6 \mathrm{mmol})$ in toluene $(0.5 \mathrm{ml})$ was heated to $120^{\circ} \mathrm{C}$. The crude product was purified by flash chromatography (30:70 petroleum ether:diethyl ether) to yield 2-ethoxy-3,4,5-tri(methoxycarbonyl)furan 3d as a white solid (0.089 g, 62 \%). m.p. 69-71 ${ }^{\circ} \mathrm{C}$; $v_{\max }(\mathrm{KBr} / \mathrm{film}) 2093 \mathrm{w}, 2958 \mathrm{~m}, 1730 \mathrm{~s}(\mathrm{C}=\mathrm{O})$, 1712 s (C=O), 1583 s, 1466 s, 1440 s, 1325 s, 1256 s, 1234 s, 1163 m, 1099 s, 1064 s, 809 w, 
$818 \mathrm{w}, 785 \mathrm{~m} \mathrm{~cm}^{-1} ; \delta_{\mathrm{H}}\left(300 \mathrm{MHz}, \mathrm{CDCl}_{3}\right) 1.45\left(3 \mathrm{H}, \mathrm{t}, J=7 \mathrm{~Hz}, \mathrm{OCH}_{2} \underline{\mathrm{CH}}_{3}\right), 3.74(3 \mathrm{H}, \mathrm{s}$, $\mathrm{CO}_{2} \mathrm{CH}_{3}$ ), 3.79 (3 H, s, $\left.\mathrm{CO}_{2} \mathrm{CH}_{3}\right), 3.89\left(3 \mathrm{H}, \mathrm{s}, \mathrm{CO}_{2} \mathrm{CH}_{3}\right), 4.55\left(2 \mathrm{H}, \mathrm{q}, J=7 \mathrm{~Hz}, \mathrm{OCH}_{2} \mathrm{CH}_{3}\right) ; \delta_{\mathrm{C}}$ (75 MHz, $\mathrm{CDCl}_{3}$ ) $14.6 \mathrm{OCH}_{2} \underline{\mathrm{CH}}_{3}, 51.7 \mathrm{CO}_{2} \underline{\mathrm{CH}_{3}}, 52.1 \mathrm{CO}_{2} \underline{\mathrm{CH}}_{3}, 52.9 \mathrm{CO}_{2} \underline{\mathrm{CH}_{3}}, 68.9 \mathrm{OCH}_{2} \mathrm{CH}_{3}$,

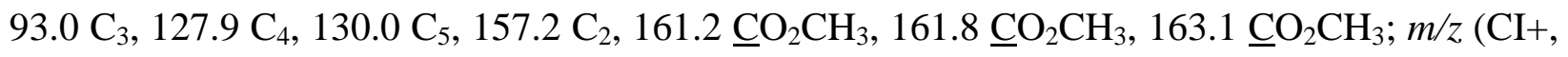
$\left.\mathrm{NH}_{3}\right) 287$ ([M+H] $\left.]^{+}, 15\right), 255$ (98), 198 (100), 140 (29), 111 (28), 49 (52); HRMS (CI+) Found $[\mathrm{M}+\mathrm{H}]^{+}$287.0757; $\mathrm{C}_{12} \mathrm{H}_{15} \mathrm{O}_{8}$ requires 287.0767.

\section{2-(2,3-Di(methoxycarbonyl)-oxabicyclo[2.2.1]hepta-2,5-dienyl)-5-ethoxy-4-triethylsilyloxazole}

(4). 5-Ethoxy-2-(2-furanyl)-4-triethylsilyloxazole $(0.034 \mathrm{~g}, 0.115 \mathrm{mmol})$ and dimethyl acetylenedicarboxylate $(16 \mu \mathrm{l}, 0.126 \mathrm{mmol})$ in toluene $(0.2 \mathrm{ml})$ was heated to $110^{\circ} \mathrm{C}$. The crude product was purified by flash chromatography (70:30 petroleum ether:diethyl ether) to yield 2(2,3-di(methoxycarbonyl)-oxabicyclo[2.2.1]hepta-2,5-dienyl)-5-ethoxy-4-triethylsilyloxazole 4 as a colourless oil (0.021 g, 43 \%). $v_{\max }(\mathrm{KBr} / \mathrm{film}) 2954 \mathrm{~s}, 2911 \mathrm{~m}, 2876 \mathrm{~m}, 1722 \mathrm{~s}(\mathrm{C}=\mathrm{O}), 1593$ s, $1437 \mathrm{~m}, 1266 \mathrm{~m}, 1117 \mathrm{~m}, 1015 \mathrm{~m}, 945 \mathrm{w}, 710 \mathrm{~m} \mathrm{~cm}^{-1}$; $\delta_{\mathrm{H}}\left(270 \mathrm{MHz}, \mathrm{CDCl}_{3}\right) 0.73(6 \mathrm{H}, \mathrm{q}, J=$ $\left.8 \mathrm{~Hz}, \mathrm{SiCH}_{2} \mathrm{CH}_{3}\right), 0.94\left(9 \mathrm{H}, \mathrm{t}, J=8 \mathrm{~Hz}, \mathrm{SiCH}_{2} \underline{\mathrm{CH}}_{3}\right), 1.34$ (3 H, t, $\left.J=7 \mathrm{~Hz}, \mathrm{OCH}_{2} \underline{\mathrm{CH}}_{3}\right), 3.73$ (3 $\mathrm{H}, \mathrm{s}, \mathrm{CO}_{2} \mathrm{CH}_{3}$ ), 3.79 (3 H, s, $\left.\mathrm{CO}_{2} \mathrm{CH}_{3}\right), 4.19$ (2 H, q, $\left.J=7 \mathrm{~Hz}, \mathrm{OCH}_{2} \mathrm{CH}_{3}\right), 5.77\left(1 \mathrm{H}, \mathrm{d}, J_{4}{ }^{\prime}-5,=2\right.$ $\left.\mathrm{Hz}, \mathrm{H}_{4}{ }^{\prime}\right), 7.29$ (1 H, dd, $J_{5^{\prime}-6^{\prime}}$ = $5 \mathrm{~Hz}, J_{5^{\prime}-4}{ }^{\prime}=2 \mathrm{~Hz}, \mathrm{H}_{5}$ ) $) 7.46\left(1 \mathrm{H}, \mathrm{d}, J_{6^{\prime}-5},=5 \mathrm{~Hz}, \mathrm{H}_{6}{ }^{\prime}\right) ; \delta_{\mathrm{C}}(75$ $\mathrm{MHz}, \mathrm{CDCl}_{3}$ ) $3.2 \mathrm{SiCH}_{2} \mathrm{CH}_{3}, 7.3 \mathrm{SiCH}_{2} \underline{\mathrm{CH}}_{3}, 14.9 \mathrm{OCH}_{2} \underline{\mathrm{CH}}_{3}, 52.3 \mathrm{CO}_{2} \underline{\mathrm{CH}}_{3}, 52.4 \mathrm{CO}_{2} \underline{\mathrm{CH}}_{3}, 69.3$ $\mathrm{OCH}_{2} \mathrm{CH}_{3}, 84.7 \mathrm{C}_{4}, 91.3 \mathrm{C}_{1}$, $109.3 \mathrm{C}_{4}, 142.7,144.3 \mathrm{C}_{5}, 6$, 148.5 , $150.8 \mathrm{C}_{2}^{\prime}, 3^{\prime}, 154.2 \mathrm{C}_{2}, 162.4$, 163.7, $165.4 \mathrm{C}_{5}, \mathrm{CO}_{2} \mathrm{CH}_{3} ; \mathrm{m} / \mathrm{z}\left(\mathrm{CI}+, \mathrm{NH}_{3}\right) 436\left([\mathrm{M}+\mathrm{H}]^{+}, 100\right) ; \mathrm{HRMS}(\mathrm{CI}+)$ Found $[\mathrm{M}+\mathrm{H}]^{+}$ 436.1794; $\mathrm{C}_{21} \mathrm{H}_{30} \mathrm{NO}_{7} \mathrm{Si}$ requires 436.1792 .

2-Ethoxy-3-methoxycarbonyl-5-phenylfuran (5a). 5-Ethoxy-2-phenyl-4-triethylsilyloxazole (0.091 g, $0.3 \mathrm{mmol})$ and methyl propiolate $(32 \mu \mathrm{l}, 0.36 \mathrm{mmol})$ in DCB $(0.3 \mathrm{ml})$ was heated to $170^{\circ} \mathrm{C}$. The crude product was purified by flash chromatography (80:20 petroleum ether:diethyl ether) to yield 2-ethoxy-3-methoxycarbonyl-5-phenylfuran 5a as a white solid (0.032 g, 43 \%). $v_{\max }(\mathrm{KBr} / \mathrm{film}) 3108 \mathrm{w}, 2910 \mathrm{w}, 1705 \mathrm{~s}(\mathrm{C}=\mathrm{O}), 1597 \mathrm{~s}, 1567 \mathrm{~m}, 1463 \mathrm{~s}, 1378 \mathrm{~s}, 1250 \mathrm{~s}, 1030 \mathrm{~m}$, $894 \mathrm{w}, 754 \mathrm{w} \mathrm{cm}^{-1}$; $\delta_{\mathrm{H}}\left(270 \mathrm{MHz}, \mathrm{CDCl}_{3}\right) 1.50\left(3 \mathrm{H}, \mathrm{t}, J=7 \mathrm{~Hz}, \mathrm{OCH}_{2} \underline{\mathrm{CH}}_{3}\right), 3.81(3 \mathrm{H}, \mathrm{s}$, $\left.\mathrm{CO}_{2} \mathrm{CH}_{3}\right), 4.54\left(2 \mathrm{H}, \mathrm{q}, J=7 \mathrm{~Hz}, \mathrm{OCH}_{2} \mathrm{CH}_{3}\right), 6.83\left(1 \mathrm{H}, \mathrm{s}, \mathrm{H}_{4}\right), 7.21\left(1 \mathrm{H}, \mathrm{m}, \mathrm{H}_{4}\right.$ ), 7.35 (2 H, m, $\mathrm{H}_{3}$ ), $7.51\left(2 \mathrm{H}, \mathrm{m}, \mathrm{H}_{2}\right.$ ); $\delta_{\mathrm{C}}\left(75 \mathrm{MHz}, \mathrm{CDCl}_{3}\right) 15.0 \mathrm{OCH}_{2} \underline{\mathrm{CH}_{3}}, 51.3 \mathrm{CO}_{2} \underline{\mathrm{CH}_{3}} 3,68.0 \mathrm{OCH}_{2} \mathrm{CH}_{3}$, 93.7 $\mathrm{C}_{3}, 106.1 \mathrm{C}_{4}, 122.8,128.7 \mathrm{C}_{2}, 33^{\prime}, 127.1 \mathrm{C}_{4}, 129.7 \mathrm{C}_{1}, 143.4 \mathrm{C}_{5}, 161.3 \mathrm{C}=\mathrm{O}, 163.4 \mathrm{C}_{2} ; \mathrm{m} / \mathrm{z}$ $\left(\mathrm{CI}+, \mathrm{NH}_{3}\right) 264\left(\left[\mathrm{M}+\mathrm{NH}_{4}\right]^{+}, 47\right), 247\left([\mathrm{M}+\mathrm{H}]^{+}, 100\right)$; HRMS $(\mathrm{CI}+)$ Found $[\mathrm{M}+\mathrm{H}]^{+} 247.0973$; $\mathrm{C}_{14} \mathrm{H}_{15} \mathrm{O}_{4}$ requires 247.0970.

2-Ethoxy-5-ethyl-3-methoxycarbonylfuran (5b). 5-Ethoxy-2-ethyl-4-triethylsilyloxazole $(0.102 \mathrm{~g}, 0.4 \mathrm{mmol})$ and methyl propiolate $(43 \mu \mathrm{l}, 0.48 \mathrm{mmol})$ in toluene $(0.4 \mathrm{ml})$ was heated to $110^{\circ} \mathrm{C}$. The crude product was purified by flash chromatography (80:20 petroleum ether:diethyl ether) to yield 2-ethoxy-3-methoxy carbonyl-5-ethylfuran $5 \mathbf{b}$ as colourless crystals (0.032 g, 40 \%). m.p. 36-37 C; $v_{\max }(\mathrm{KBr} /$ film) 3125 w, 2976 s, 2955 s, 2879 m, 1719 s (C=O), 1604 s, 1458 m, 1416 w, 1388 w, 1363 w, 1248 m, 1197 m, 1138 m, 1087 s, 1020 m, 960 w, 890 w, 818 w, $776 \mathrm{~m}, 741 \mathrm{w} \mathrm{cm}^{-1}$; $\delta_{\mathrm{H}}\left(270 \mathrm{MHz}, \mathrm{CDCl}_{3}\right) 1.16\left(3 \mathrm{H}, \mathrm{t}, J=7.5 \mathrm{~Hz}, \underline{\mathrm{CH}}_{3} \mathrm{CH}_{2}\right), 1.42(3 \mathrm{H}, \mathrm{t}, J=$ $7 \mathrm{~Hz}, \mathrm{OCH}_{2} \underline{\mathrm{CH}}_{3}$ ), 2.50 (2 H, dq, $J=1 \mathrm{~Hz}, J=7.5 \mathrm{~Hz}, \mathrm{CH}_{3} \underline{\mathrm{CH}}_{2}$ ), 3.75 (3 H, s, $\mathrm{CO}_{2} \mathrm{CH}_{3}$ ), 4.39 (2 $\left.\mathrm{H}, \mathrm{q}, J=7 \mathrm{~Hz}, \underline{\mathrm{OCH}}_{2} \mathrm{CH}_{3}\right), 6.14\left(1 \mathrm{H}, \mathrm{t}, J=1 \mathrm{~Hz}, \mathrm{H}_{4}\right) ; \delta_{\mathrm{C}}\left(75 \mathrm{MHz}, \mathrm{CDCl}_{3}\right) 11.6 \underline{\mathrm{CH}_{3} \mathrm{CH}_{2}, 15.0}$ 
$\mathrm{OCH}_{2} \underline{\mathrm{CH}_{3}}, 20.9 \mathrm{CH}_{3} \underline{\mathrm{CH}}_{2}, 51.0 \mathrm{CO}_{2} \underline{\mathrm{CH}_{3}}, 67.1 \underline{\mathrm{OCH}}_{2} \mathrm{CH}_{3}, 92.1 \mathrm{C}_{3}, 104.9 \mathrm{C}_{4}, 147.3 \mathrm{C}_{5}, 160.8 \mathrm{C}_{2}$, $163.7 \mathrm{CO}_{2} \mathrm{CH}_{3} ; \mathrm{m} / \mathrm{z}\left(\mathrm{CI}+, \mathrm{NH}_{3}\right) 216\left(\left[\mathrm{M}+\mathrm{NH}_{4}\right]^{+}, 22\right), 199\left([\mathrm{M}+\mathrm{H}]^{+}, 46\right), 102$ (34), 52 (100); HRMS (CI+) Found $[\mathrm{M}+\mathrm{H}]^{+} 199.0971 ; \mathrm{C}_{10} \mathrm{H}_{15} \mathrm{O}_{4}$ requires 199.0970 .

2-(4-(tert-Butyldimethylsilyloxy)-butyl)-5-ethoxy-4-triethylsilyl oxazole (7). The experiment was carried out by the general procedure for the preparation of 4-silylated oxazoles with 5-(tertbutyldimethylsilyloxy)-pentanenitrile ( $2.77 \mathrm{~g}, 13 \mathrm{mmol}$ ), ethyl (triethylsilyl)diazoacetate (2.28 g, $10 \mathrm{mmol}$ ) and $\mathrm{Rh}_{2}$ (octanoate $)_{4}(78 \mathrm{mg}, 0.1 \mathrm{mmol})$ in dry benzene $(20 \mathrm{ml})$. The crude product was purified by flash chromatography (95:5 petroleum ether:diethyl ether) to yield 2-(4-(tertbutyldimethylsilyloxy)butyl)-5-ethoxy-4-triethylsilyloxazole 7 as a colourless liquid (3.30 g, 79 \%). $v_{\max }(\mathrm{KBr} /$ film) 2953 s, 2934 s, 2874 s, 1605 s, 1577 w, 1462 w, 1389 w, 1255 m, 1105 m, 1019 m, 836 m, 775 w, 736 m, $734 \mathrm{~m} \mathrm{~cm}^{-1}$; $\delta_{\mathrm{H}}\left(270 \mathrm{MHz}, \mathrm{CDCl}_{3}\right) 0.02\left(6 \mathrm{H}, \mathrm{s}, \mathrm{SiCH}_{3}\right), 0.72$ $\left(6 \mathrm{H}, \mathrm{q}, J=8 \mathrm{~Hz}, \mathrm{SiCH}_{2} \mathrm{CH}_{3}\right), 0.86\left(9 \mathrm{H}, \mathrm{s}, \mathrm{SiC}\left(\underline{\mathrm{CH}}_{3}\right)_{3}\right), 0.94\left(9 \mathrm{H}, \mathrm{t}, J=8 \mathrm{~Hz}, \mathrm{SiCH}_{2} \underline{\mathrm{CH}}_{3}\right), 1.33$ ( $3 \mathrm{H}, \mathrm{t}, J=7 \mathrm{~Hz}, \mathrm{OCH}_{2} \underline{\mathrm{CH}}_{3}$ ), 1.55 (2 H, p, $J=7 \mathrm{~Hz}, \mathrm{H}_{3}$ ), 1.73 (2 H, p, $J=7 \mathrm{~Hz}, \mathrm{H}_{2}$ ), 2.64 (2 H, t, $J_{1^{\prime}-2^{\prime}}=7 \mathrm{~Hz}, \mathrm{H}_{1^{\prime}}$ ), $3.60\left(2 \mathrm{H}, \mathrm{t}, J_{4^{\prime}-3^{\prime}}=6.5 \mathrm{~Hz}, \mathrm{H}_{4}{ }^{\prime}\right), 4.12\left(2 \mathrm{H}, \mathrm{q}, J=7 \mathrm{~Hz}, \mathrm{OCH}_{2} \mathrm{CH}_{3}\right) ; \delta_{\mathrm{C}}(75$ $\left.\mathrm{MHz}, \mathrm{CDCl}_{3}\right)-5.4 \mathrm{SiCH}_{3}, 3.1 \mathrm{SiCH}_{2} \mathrm{CH}_{3}, 7.3 \mathrm{SiCH}_{2} \underline{\mathrm{CH}}_{3}, 14.9 \mathrm{OCH}_{2} \underline{\mathrm{CH}}_{3}, 18.3 \mathrm{SiC}\left(\mathrm{CH}_{3}\right)_{3}, 23.7$ $\mathrm{C}_{2}$, $25.9 \mathrm{SiC}\left(\underline{\mathrm{CH}}_{3}\right)_{3}, 28.3 \mathrm{C}_{1}$, $32.2 \mathrm{C}_{3}$, $62.7 \mathrm{C}_{4}, 69.2 \mathrm{OCH}_{2} \mathrm{CH}_{3}, 109.4 \mathrm{C}_{4}, 157.1 \mathrm{C}_{2}, 164.5 \mathrm{C}_{5}$; $\mathrm{m} / \mathrm{z}\left(\mathrm{CI}+, \mathrm{NH}_{3}\right) 414\left([\mathrm{M}+\mathrm{H}]^{+}, 100\right), 356$ (10), 208 (10), $132\left(\left[\mathrm{SiEt}_{3}+\mathrm{NH}_{3}\right]^{+}, 5\right)$; HRMS (CI+) Found $[\mathrm{M}+\mathrm{H}]^{+} 414.2854 ; \mathrm{C}_{21} \mathrm{H}_{44} \mathrm{NO}_{3} \mathrm{Si}_{2}$ requires 414.2860 .

5-Ethoxy- 2-(4-hydroxybutyl)-4-triethylsilyloxazole (8). To a stirred solution of 2-(4-(tertbutyldimethylsilyloxy)butyl)-5-ethoxy-4-triethylsilyl oxazole 7 (3.20 g, $7.73 \mathrm{mmol}$ ) in THF $(30 \mathrm{ml})$ was added TBAF $(7.73 \mathrm{ml}$ of a $1 \mathrm{M}$ solution in THF, $7.73 \mathrm{mmol})$. The mixture was stirred at rt and monitored by TLC. After complete consumption of the starting material, the reaction was quenched by the addition of sat. aq. $\mathrm{NH}_{4} \mathrm{Cl}(30 \mathrm{ml})$. The aqueous layer was extracted with ether $(3 \times 30 \mathrm{ml})$ and the combined organics dried $\left(\mathrm{MgSO}_{4}\right)$, filtered and evaporated under reduced pressure. The crude product was purified by flash chromatography (10:90 petroleum ether:diethyl ether) to yield 2-(4-hydroxybutyl)-5-ethoxy-4-triethylsilyloxazole 8 as a colourless oil (2.10 g, $91 \%)$. $v_{\max }(\mathrm{KBr} / \mathrm{film}) 3343 \mathrm{~m}(\mathrm{OH}), 2954$ s, 2933 s, 2872 s, 1606 m, 1512 w, 1465 w, 1377 w, 1246 m, 1067 m, 1017 m, 739 w cm'; $\delta_{\mathrm{H}}\left(270 \mathrm{MHz}, \mathrm{CDCl}_{3}\right) 0.70$ $\left(6 \mathrm{H}, \mathrm{q}, J=8 \mathrm{~Hz}, \mathrm{SiCH}_{2} \mathrm{CH}_{3}\right), 0.92\left(9 \mathrm{H}, \mathrm{t}, J=8 \mathrm{~Hz}, \mathrm{SiCH}_{2} \underline{\mathrm{CH}}_{3}\right), 1.33(3 \mathrm{H}, \mathrm{t}, J=7 \mathrm{~Hz}$, $\left.\mathrm{OCH}_{2} \underline{\mathrm{CH}}_{3}\right), 1.60$ (2 H, p, $\left.J=7 \mathrm{~Hz}, \mathrm{H}_{3}{ }^{\prime}\right), 1.81$ (2 H, p, $J_{2^{\prime}-1}{ }^{\prime}=J_{2^{\prime}-3^{\prime}}=7 \mathrm{~Hz}, \mathrm{H}_{2}$ ), 2.66 (2 H, t, $J_{1^{\prime}-2^{\prime}}$ $=7 \mathrm{~Hz}, \mathrm{H}_{1}$ ), $2.95\left(1 \mathrm{H}\right.$, br s, OH), $3.60\left(2 \mathrm{H}, \mathrm{t}, J_{4^{\prime}-3^{\prime}}=6 \mathrm{~Hz}, \mathrm{H}_{4}{ }^{\prime}\right), 4.12(2 \mathrm{H}, \mathrm{q}, J=7 \mathrm{~Hz}$, $\left.\mathrm{OCH}_{2} \mathrm{CH}_{3}\right)\left(\mathrm{OH}\right.$ not observed); $\delta_{\mathrm{C}}\left(67.5 \mathrm{MHz}, \mathrm{CDCl}_{3}\right) 3.1 \mathrm{SiCH}_{2} \mathrm{CH}_{3}, 7.3 \mathrm{SiCH}_{2} \underline{\mathrm{CH}} 3,14.9$ $\mathrm{OCH}_{2} \underline{\mathrm{CH}_{3}}, 22.5 \mathrm{C}_{2}$, $27.8 \mathrm{C}_{1}$, $32.1 \mathrm{C}_{3}$, $61.5 \mathrm{C}_{4}, 69.5 \mathrm{OCH}_{2} \mathrm{CH}_{3}, 99.9 \mathrm{C}_{4}, 157.4 \mathrm{C}_{2}, 164.5 \mathrm{C}_{5}$; $\mathrm{m} / \mathrm{z}\left(\mathrm{CI}+, \mathrm{NH}_{3}\right) 338\left(\left[\mathrm{M}+2 \mathrm{H}+2 \mathrm{NH}_{4}\right]^{+}, 14\right), 300\left([\mathrm{M}+\mathrm{H}]^{+}, 100\right) ; \mathrm{HRMS}(\mathrm{CI}+)$ Found $[\mathrm{M}+\mathrm{H}]^{+}$ 300.1992; $\mathrm{C}_{15} \mathrm{H}_{30} \mathrm{NO}_{3} \mathrm{Si}$ requires 300.1995.

5-Ethoxy-2-(4-oxobutyl)-4-triethylsilyloxazole (9). To a stirred solution of oxalyl chloride (0.95 g, $7.5 \mathrm{mmol})$ in dry DCM $(30 \mathrm{ml})$ cooled to $-78^{\circ} \mathrm{C}$ was added dropwise DMSO (1.06 ml, $15 \mathrm{mmol}$ ). The mixture was stirred for $5 \mathrm{~min}$ at $-78^{\circ} \mathrm{C}$ and a solution of 2-(4-hydroxybutyl)-5ethoxy-4-triethylsilyloxazole 8 (1.50 g, $5 \mathrm{mmol})$ in dry DCM (30 ml) was added in one portion. The reaction mixture was kept at $-78^{\circ} \mathrm{C}$ for $30 \mathrm{~min}$ and was then treated with $\mathrm{Et}_{3} \mathrm{~N}(4.2 \mathrm{ml}$, 
$30 \mathrm{mmol}$ ). After an additional $10 \mathrm{~min}$ at $-78^{\circ} \mathrm{C}$ the mixture was allowed to warm slowly to rt. $\mathrm{H}_{2} \mathrm{O}(20 \mathrm{ml})$ was added and the organic phase was separated. The aqueous phase was washed with DCM (2 x $20 \mathrm{ml})$ and the combined organics were washed with saturated brine $(20 \mathrm{ml})$, dried $\left(\mathrm{MgSO}_{4}\right)$, filtered and evaporated under reduced pressure. The crude product was purified by flash chromatography (40:60 petroleum ether:diethyl ether) to yield 5-ethoxy-2-(4-oxobutyl)4-triethylsilyloxazole 9 as a colourless oil (1.09 g, $73 \%) \cdot v_{\max }(\mathrm{KBr} / \mathrm{film}) 2954 \mathrm{~s}, 2934 \mathrm{~s}, 2874 \mathrm{~s}$, $1725 \mathrm{~s}(\mathrm{C}=\mathrm{O}), 1605 \mathrm{~s}, 1576 \mathrm{w}, 1512 \mathrm{w}, 1460 \mathrm{w}, 1390 \mathrm{~m}, 1242 \mathrm{~m}, 1018 \mathrm{~m}, 737 \mathrm{~m}, 724 \mathrm{~m} \mathrm{~cm}^{-1}$; $\delta_{\mathrm{H}}\left(300 \mathrm{MHz}, \mathrm{CDCl}_{3}\right) 0.71\left(6 \mathrm{H}, \mathrm{q}, J=8 \mathrm{~Hz}, \mathrm{SiCH}_{2} \mathrm{CH}_{3}\right), 0.93\left(9 \mathrm{H}, \mathrm{t}, J=8 \mathrm{~Hz}, \mathrm{SiCH}_{2} \mathrm{CH}_{3}\right)$, $1.33\left(3 \mathrm{H}, \mathrm{t}, J=7 \mathrm{~Hz}, \mathrm{OCH}_{2} \mathrm{CH}_{3}\right), 2.01\left(2 \mathrm{H}, \mathrm{p}, J_{2^{\prime}-1}{ }^{\prime}=J_{2^{\prime}-3^{\prime}}=7 \mathrm{~Hz}, \mathrm{H}_{2^{\prime}}\right), 2.51\left(2 \mathrm{H}, \mathrm{dt}, J_{3^{\prime}-4^{\prime}}=1\right.$ $\left.\mathrm{Hz}, J_{3^{\prime}-2^{\prime}}=7 \mathrm{~Hz}, \mathrm{H}_{3^{\prime}}\right), 2.68\left(2 \mathrm{H}, \mathrm{t}, J_{1^{\prime}-2^{\prime}}=7 \mathrm{~Hz}, \mathrm{H}_{1^{\prime}}\right), 4.13\left(2 \mathrm{H}, \mathrm{q}, J=7 \mathrm{~Hz}, \mathrm{OCH}_{2} \mathrm{CH}_{3}\right), 9.74(1$ $\left.\mathrm{H}, \mathrm{t}, J_{4^{\prime}-3^{\prime}}=1 \mathrm{~Hz}, \mathrm{H}_{4}{ }^{\prime}\right) ; \delta_{\mathrm{C}}\left(75 \mathrm{MHz}, \mathrm{CDCl}_{3}\right) 3.1 \mathrm{SiCH}_{2} \mathrm{CH}_{3}, 7.3 \mathrm{SiCH}_{2} \mathrm{CH}_{3}, 14.9 \mathrm{OCH}_{2} \mathrm{CH}_{3}, 19.7$ $\mathrm{C}_{2}$, $27.6 \mathrm{C}_{1}$, $42.9 \mathrm{C}_{3}, 69.5 \mathrm{OCH}_{2} \mathrm{CH}_{3}, 109.7 \mathrm{C}_{4}, 156.0 \mathrm{C}_{2}, 164.7 \mathrm{C}_{5}, 201.5 \mathrm{C}=\mathrm{O} ; \mathrm{m} / \mathrm{z}\left(\mathrm{CI}+, \mathrm{NH}_{3}\right)$ $298\left([\mathrm{M}+\mathrm{H}]^{+}, 100\right)$; HRMS (CI+) Found $[\mathrm{M}+\mathrm{H}]^{+}$298.1845; $\mathrm{C}_{15} \mathrm{H}_{28} \mathrm{NO}_{3} \mathrm{Si}$, requires 298.1838.

5-Ethoxy-2-(4-hydroxy-6-methoxycarbonyl-hex-5-ynyl)-4-triethylsilyloxazole (10a). A solution of methyl propiolate $(0.29 \mathrm{ml}, 3.26 \mathrm{mmol})$ in $4: 1: 1$ dry $\mathrm{THF} / \mathrm{Et}_{2} \mathrm{O} /$ pentane $(20 \mathrm{ml}$ total) was cooled to $-120^{\circ} \mathrm{C}$ (the cooling bath consisted of a $4: 1: 1$ mixture of low boiling petroleum ether/acetone/isopropyl alcohol cooled with liquid nitrogen). $n$-BuLi (2.04 ml of a 1.6 M solution in THF, $3.26 \mathrm{mmol}$ ) was added dropwise, with vigorous stirring, over a period of 15 min while maintaining a temperature of $-120^{\circ} \mathrm{C}$. Stirring was continued for $15 \mathrm{~min}$ at $-120^{\circ} \mathrm{C}$ to complete the formation of lithiated methyl propiolate. A solution of 5-ethoxy-2-(4-oxobutyl)-4triethylsilyloxazole 9 (0.776 g, $2.6 \mathrm{mmol}$ ) in 4:1:1 dry THF/Et $2 \mathrm{O} /$ pentane (20 ml total) was then added dropwise with vigorous stirring. The mixture was stirred for $15 \mathrm{~min}$ at $-120^{\circ} \mathrm{C}$ and the cooling bath was removed. After warming to $-78^{\circ} \mathrm{C}$, the reaction mixture was quenched with a $10 \%$ aqueous solution of $\mathrm{KH}_{2} \mathrm{PO}_{4}(20 \mathrm{ml})$, washed with $\mathrm{H}_{2} \mathrm{O}(20 \mathrm{ml})$ and extracted with $\mathrm{Et}_{2} \mathrm{O}(3$ x $20 \mathrm{ml})$. The combined organics were dried $\left(\mathrm{MgSO}_{4}\right)$, filtered and evaporated under reduced pressure to afford a yellow oil. The crude product was purified by flash chromatography (30:70 petroleum ether:diethyl ether) to yield 2-(4-hydroxy-6-methoxycarbonyl-hex-5-ynyl)-5-ethoxy4-triethylsilyloxazole as a colourless oil (0.77 g, $78 \%$ ). $v_{\max }(\mathrm{KBr} / \mathrm{film}) 3218 \mathrm{w}(\mathrm{OH}), 2954 \mathrm{~m}$, $2912 \mathrm{w}, 2235 \mathrm{w}(\mathrm{C} \equiv \mathrm{C}), 1720 \mathrm{~s}(\mathrm{C}=\mathrm{O}), 1606 \mathrm{~s}, 1434 \mathrm{w}, 1249 \mathrm{~s}, 1016 \mathrm{w}, 738 \mathrm{w}, 723 \mathrm{w} \mathrm{cm}^{-1} ; \delta_{\mathrm{H}}$ $\left(270 \mathrm{MHz}, \mathrm{CDCl}_{3}\right) 0.71\left(6 \mathrm{H}, \mathrm{q}, J=8 \mathrm{~Hz}, \mathrm{SiCH}_{2} \mathrm{CH}_{3}\right) 0.93\left(9 \mathrm{H}, \mathrm{t}, J=8 \mathrm{~Hz}, \mathrm{SiCH}_{2} \mathrm{CH}_{3}\right), 1.34(3$ $\left.\mathrm{H}, \mathrm{t}, J=7 \mathrm{~Hz}, \mathrm{OCH}_{2} \underline{\mathrm{CH}}_{3}\right), 1.80-2.05\left(4 \mathrm{H}, \mathrm{m}, \mathrm{H}_{2^{\prime}, 3^{\prime}}\right), 2.60-2.82\left(2 \mathrm{H}, \mathrm{m}, \mathrm{H}_{1^{\prime}}\right), 3.74(3 \mathrm{H}, \mathrm{s}$, $\left.\mathrm{CO}_{2} \mathrm{CH}_{3}\right), 4.13\left(2 \mathrm{H}, \mathrm{q}, J=7 \mathrm{~Hz}, \mathrm{OCH}_{2} \mathrm{CH}_{3}\right), 4.50\left(1 \mathrm{H}, \mathrm{t}, J_{4^{\prime}-3}{ }^{\prime}=6 \mathrm{~Hz}, \mathrm{H}_{4}{ }^{\prime}\right)(\mathrm{OH}$ not observed); $\delta_{\mathrm{C}}\left(75 \mathrm{MHz}, \mathrm{CDCl}_{3}\right) 3.1 \mathrm{SiCH}_{2} \mathrm{CH}_{3}, 7.3 \mathrm{SiCH}_{2} \underline{\mathrm{CH}}_{3}, 15.0 \mathrm{OCH}_{2} \underline{\mathrm{CH}}_{3}, 21.0 \mathrm{C}_{2}, 27.4 \mathrm{C}_{1}, 36.1 \mathrm{C}_{3}$, $52.7 \mathrm{CO}_{2} \underline{\mathrm{CH}} 3,60.8 \mathrm{C}_{4}, 69.5 \mathrm{OCH}_{2} \mathrm{CH}_{3}, 75.7 \mathrm{C}_{6}, 88.5 \mathrm{C}_{5}, 109.3 \mathrm{C}_{4}, 153.8 \mathrm{C}_{2}, 157.0 \mathrm{C}_{5}, 164.6$ $\mathrm{C}=\mathrm{O} ; \mathrm{m} / \mathrm{z}\left(\mathrm{CI}+, \mathrm{NH}_{3}\right) 382\left([\mathrm{M}+\mathrm{H}]^{+}, 100\right)$; HRMS (CI+) Found $[\mathrm{M}+\mathrm{H}]^{+}$382.2057; $\mathrm{C}_{19} \mathrm{H}_{32} \mathrm{NO}_{5} \mathrm{Si}$, requires 382.2050.

5-Ethoxy-2-(4-hydroxy-6-trimethylsilylhex-5-ynyl)-4-triethylsilyloxazole (10b). To a solution of (trimethylsilyl)acetylene ( $64 \mu \mathrm{l}, 0.45 \mathrm{mmol})$ in THF $(2 \mathrm{ml})$ cooled to $-78^{\circ} \mathrm{C}$ was added dropwise $n$-BuLi $(0.28 \mathrm{ml}$ of a $1.6 \mathrm{M}$ solution in THF, $0.45 \mathrm{mmol})$. Stirring was continued for $15 \mathrm{~min}$ at $-78^{\circ} \mathrm{C}$ and a solution of 5-ethoxy-2-(4-oxobutyl)-4-triethylsilyloxazole 9 (0.089 g, 
$0.30 \mathrm{mmol})$ in THF $(2 \mathrm{ml})$ was added in one portion. The reaction mixture was stirred for $15 \mathrm{~min}$ at $-78^{\circ} \mathrm{C}$ and then was quenched with a $10 \%$ aqueous solution of $\mathrm{KH}_{2} \mathrm{PO}_{4}(5 \mathrm{ml})$, washed with $\mathrm{H}_{2} \mathrm{O}(5 \mathrm{ml})$ and extracted with $\mathrm{Et}_{2} \mathrm{O}(3 \mathrm{x} 5 \mathrm{ml})$. The combined organics were dried $\left(\mathrm{MgSO}_{4}\right)$, filtered and evaporated under reduced pressure to afford a yellow oil. The crude product was purified by flash chromatography (60:40 petroleum ether:diethyl ether) to yield 5-ethoxy-2-(4hydroxy-6-trimethylsilylhex-5-ynyl)-4-triethylsilyloxazole as a colourless oil (0.107 g, 90 \%). $v_{\max }(\mathrm{KBr} / \mathrm{film}) 3275 \mathrm{~s}(\mathrm{OH}), 2953 \mathrm{~s}, 2874 \mathrm{~s}, 2170 \mathrm{w}(\mathrm{C} \equiv \mathrm{C}), 1727 \mathrm{~m}, 1606 \mathrm{~s}, 1574 \mathrm{~m}, 1458 \mathrm{~m}$, 1414 m, 1391 m, 1249 s, 1132 m, 1076 m, 1017 s, 842 s, 737 s, 725 s cm ${ }^{-1}$; $\delta_{\mathrm{H}}(270 \mathrm{MHz}$, $\left.\mathrm{CDCl}_{3}\right) 0.13\left(9 \mathrm{H}, \mathrm{s}, \mathrm{SiCH}_{3}\right), 0.72\left(6 \mathrm{H}, \mathrm{q}, J=8 \mathrm{~Hz}, \mathrm{SiCH}_{2} \mathrm{CH}_{3}\right), 0.93(9 \mathrm{H}, \mathrm{t}, J=8 \mathrm{~Hz}$, $\left.\mathrm{SiCH}_{2} \underline{\mathrm{CH}}_{3}\right), 1.34$ (3 H, t, $\left.J=7 \mathrm{~Hz}, \mathrm{OCH}_{2} \underline{\mathrm{CH}}_{3}\right), 1.71-1.80\left(2 \mathrm{H}, \mathrm{m}, \mathrm{H}_{2}\right), 1.88$ (2 H, appar q, $J=$ $7 \mathrm{~Hz}, \mathrm{H}_{1}$ ), 2.69 (2 H, app q, $J=7 \mathrm{~Hz}, \mathrm{H}_{3}$ ), 3.06 (1 H, br s, OH), 4.13 (2 H, $J=7 \mathrm{~Hz}, \mathrm{q}$, $\left.\mathrm{OCH}_{2} \mathrm{CH}_{3}\right), 4.37\left(1 \mathrm{H}, \mathrm{t}, J_{4^{\prime}-3}{ }^{\prime}=6.5 \mathrm{~Hz}, \mathrm{H}_{4}{ }^{\prime}\right), \delta_{\mathrm{C}}\left(75 \mathrm{MHz}, \mathrm{CDCl}_{3}\right)-0.2 \mathrm{SiCH}_{3}, 3.0 \mathrm{SiCH}_{2} \mathrm{CH}_{3}$, 7.2 $\mathrm{SiCH}_{2} \underline{\mathrm{CH}}_{3}, 14.9 \mathrm{OCH}_{2} \underline{\mathrm{CH}}_{3}, 22.2 \mathrm{C}_{2}, 27.7 \mathrm{C}_{1}$, $36.9 \mathrm{C}_{3}, 61.6 \mathrm{C}_{4}, 69.3 \mathrm{OCH}_{2} \mathrm{CH}_{3}, 88.6 \mathrm{C}_{6}$, 106.9 $\mathrm{C}_{5}, 109.2 \mathrm{C}_{4}, 157.1 \mathrm{C}_{2}, 164.4 \mathrm{C}_{5} ; \mathrm{m} / \mathrm{z}\left(\mathrm{CI}+, \mathrm{NH}_{3}\right) 396\left([\mathrm{M}+\mathrm{H}]^{+}, 100\right), 298$ (15); HRMS $(\mathrm{CI}+)$ Found $[\mathrm{M}+\mathrm{H}]^{+}$396.2398; $\mathrm{C}_{20} \mathrm{H}_{38} \mathrm{NO}_{3} \mathrm{Si}_{2}$, requires 396.2390.

5-Ethoxy-2-(4-oxo-6-methoxycarbonylhex-5-ynyl)-4-triethylsilyloxazole (6a). To a solution of 5-ethoxy-2-(4-hydroxy-6-methoxycarbonyl-hex-5-ynyl)-4-triethylsilyloxazole 10a (0.496 g, $1.3 \mathrm{mmol})$ in DCM $(8 \mathrm{ml})$ at rt was added Dess-Martin periodinane (0.652 g, $1.56 \mathrm{mmol})$. After the reaction was complete as indicated by TLC analysis, the reaction mixture was quenched by the addition of sat. aq. $\mathrm{NaHCO}_{3}(10 \mathrm{ml})$. The mixture was partitioned between DCM and sat. aq. $\mathrm{NaHCO}_{3}$. The combined organics were dried $\left(\mathrm{MgSO}_{4}\right)$, filtered and evaporated under reduced pressure to afford a pale yellow oil. The crude product was purified by flash chromatography (50:50 petroleum ether:diethyl ether) to yield 5-ethoxy-2-(4-oxo-6-methoxycarbonylhex-5-ynyl)4-triethylsilyloxazole 6a as a colourless oil (0.379 g, $77 \%$ ) which was used directly in the next step. $v_{\max }(\mathrm{KBr} / \mathrm{film}) 2954 \mathrm{~s}, 2911 \mathrm{~m}, 2876 \mathrm{~m}, 2214 \mathrm{w}(\mathrm{C} \equiv \mathrm{C}), 1726 \mathrm{~s}\left(\mathrm{CO}_{2} \mathrm{Me}\right), 1691 \mathrm{~s}(\mathrm{C}=\mathrm{O})$, 1606 s, 1435 w, 1254 s, 1115 w, 1010 m, 890 w, 737 m, $725 \mathrm{~m} \mathrm{~cm}^{-1}$; $\delta_{\mathrm{H}}\left(300 \mathrm{MHz}, \mathrm{CDCl}_{3}\right) 0.66$ $\left(6 \mathrm{H}, \mathrm{q}, J=8 \mathrm{~Hz}, \underline{\mathrm{SiCH}}_{2} \mathrm{CH}_{3}\right), 0.88\left(9 \mathrm{H}, \mathrm{t}, J=8 \mathrm{~Hz}, \mathrm{SiCH}_{2} \underline{\mathrm{CH}}_{3}\right), 1.28(3 \mathrm{H}, \mathrm{t}, J=7 \mathrm{~Hz}$,

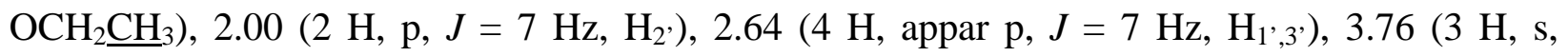
$\mathrm{CO}_{2} \mathrm{Me}$ ), $4.08\left(2 \mathrm{H}, \mathrm{q}, J=7 \mathrm{~Hz}, \mathrm{OCH}_{2} \mathrm{CH}_{3}\right) ; \delta_{\mathrm{C}}\left(75 \mathrm{MHz}, \mathrm{CDCl}_{3}\right) 3.1 \mathrm{SiCH}_{2} \mathrm{CH}_{3}, 7.3$ $\mathrm{SiCH}_{2} \underline{\mathrm{CH}}_{3}, 14.9 \mathrm{OCH}_{2} \underline{\mathrm{CH}}_{3}, 20.7 \mathrm{C}_{2}, 27.2 \mathrm{C}_{1}, 44.1 \mathrm{C}_{3}, 53.3 \mathrm{CO}_{2} \underline{\mathrm{CH}_{3}}, 69.4 \mathrm{OCH}_{2} \mathrm{CH}_{3}, 77.8 \mathrm{C}_{6}$, $80.6 \mathrm{C}_{5}, 109.7 \mathrm{C}_{4}, 152.5 \mathrm{C}_{2}, 155.5 \mathrm{C}_{5}, 164.6 \mathrm{CO}_{2} \mathrm{Me}, 184.8 \mathrm{C}_{4} ; \mathrm{m} / \mathrm{z}\left(\mathrm{CI}+, \mathrm{NH}_{3}\right) 398$ $\left(\left[\mathrm{M}+\mathrm{NH}_{4}\right]^{+}, 22\right), 380\left([\mathrm{M}+\mathrm{H}]^{+}, 73\right), 239$ (100); HRMS (CI+) Found $[\mathrm{M}+\mathrm{H}]^{+}$380.1899; $\mathrm{C}_{19} \mathrm{H}_{30} \mathrm{NO}_{5} \mathrm{Si}$, requires 380.1893 .

5-Ethoxy-2-(4-oxo-6-trimethylsilylhex-5-ynyl)-4-triethylsilyloxazole (6b). The experiment was carried out by the procedure used for the preparation of 6a with 2-(4-hydroxy-6trimethylsilylhex-5-ynyl)-5-ethoxy-4-triethylsilyloxazole $(0.112 \mathrm{~g}, 0.283 \mathrm{mmol})$ and DessMartin periodinane $(0.142 \mathrm{~g}, 0.34 \mathrm{mmol})$ in dry DCM $(2 \mathrm{ml})$. The crude product was purified by flash chromatography (60:40 petroleum ether:diethyl ether) to yield 5-ethoxy-2-(4-oxo-6trimethylsilylhex-5-ynyl)-4-triethylsilyloxazole $\mathbf{6 b}$ as a colourless liquid (0.089 g, $80 \%$ ). $v_{\max }$ (KBr/film) 2954 s, 2911 s, 2874 s, 2150 w (C =C), 1679 s (C=O), 1605 s, 1252 s, 1110 s, 1019 m, 
$847 \mathrm{~s}, 737 \mathrm{~s}, 724 \mathrm{~s} \mathrm{~cm}^{-1}$; $\delta_{\mathrm{H}}\left(300 \mathrm{MHz}, \mathrm{CDCl}_{3}\right) 0.20\left(9 \mathrm{H}, \mathrm{s}, \mathrm{SiCH}_{3}\right), 0.71(6 \mathrm{H}, \mathrm{q}, J=8 \mathrm{~Hz}$, $\mathrm{SiCH}_{2} \mathrm{CH}_{3}$ ), 0.93 (9 H, t, $\left.J=8 \mathrm{~Hz}, \mathrm{SiCH}_{2} \underline{\mathrm{CH}}_{3}\right), 1.33$ (3 H, t, $J=7 \mathrm{~Hz}, \mathrm{OCH}_{2} \underline{\mathrm{CH}}_{3}$ ), 2.02 (2 H, p, $J$ $=7 \mathrm{~Hz}, \mathrm{H}_{2}$ ), 2.62 (2 H, t, $J=7 \mathrm{~Hz}, \mathrm{H}_{1}$ ), 2.65 (2 H, t, $J=7 \mathrm{~Hz}, \mathrm{H}_{3}$ ), $4.12(2 \mathrm{H}, \mathrm{q}, J=7 \mathrm{~Hz}$, $\left.\mathrm{OCH}_{2} \mathrm{CH}_{3}\right) ; \delta_{\mathrm{C}}\left(75 \mathrm{MHz}, \mathrm{CDCl}_{3}\right)-0.8 \mathrm{SiCH}_{3}, 3.1 \mathrm{SiCH}_{2} \mathrm{CH}_{3}, 7.3, \mathrm{SiCH}_{2} \underline{\mathrm{CH}_{3}}, 14.9 \mathrm{OCH}_{2} \underline{\mathrm{CH}_{3}}$, 21.2 $\mathrm{C}_{2}$, $27.5 \mathrm{C}_{1}$, $44.2 \mathrm{C}_{3}$, $69.4 \mathrm{OCH}_{2} \mathrm{CH}_{3}, 98.0 \mathrm{C}_{6}$, $101.8 \mathrm{C}_{5}, 109.6 \mathrm{C}_{4}, 156.0 \mathrm{C}_{2}, 164.6 \mathrm{C}_{5}$, $186.7 \mathrm{C}_{4} ; \mathrm{m} / \mathrm{z}\left(\mathrm{CI}+, \mathrm{NH}_{3}\right) 394\left([\mathrm{M}+\mathrm{H}]^{+}, 100\right), 132$ ([SiEt $\left.\left.3+\mathrm{NH}_{3}\right]^{+}, 26\right), 90$ (37); HRMS (CI+) Found $[\mathrm{M}+\mathrm{H}]^{+}$394.2246; $\mathrm{C}_{20} \mathrm{H}_{36} \mathrm{NO}_{3} \mathrm{Si}$, requires 394.2234.

Preparation of 7-phenyl-hept-6-ynenitrile (11). A solution of 6-bromo-1-phenylhex-1-yne ${ }^{13}$ (1.34 g, $5.65 \mathrm{mmol})$ and $\mathrm{KCN}(1.47 \mathrm{~g}, 22.6 \mathrm{mmol})$ in acetone $(4 \mathrm{ml})$ and water $(4 \mathrm{ml})$ was heated to $65{ }^{\circ} \mathrm{C}$ for $35 \mathrm{~h}$. The reaction mixture was diluted with water $(20 \mathrm{ml})$ and extracted with ether (3 x $30 \mathrm{ml})$. The combined organics were washed with brine $(2 \times 20 \mathrm{ml})$, dried $\left(\mathrm{MgSO}_{4}\right)$, filtered and concentrated under reduced pressure. The residual oil was purified by flash chromatography (70:30 petroleum ether:diethyl ether) to yield 7-phenyl-hept-6-ynenitrile $\mathbf{1 1}$ as a colourless liquid (0.636 g, 62 \%). v $v_{\max }$ (KBr/film) 3057 w, 2934 s, 2867 m, 2245 w (CN), 1597 w, 1489 s, 1441 m, 1249 w, 1070 w, 757 s, $693 \mathrm{~s} \mathrm{~cm}^{-1}, \delta_{\mathrm{H}}\left(270 \mathrm{MHz} \mathrm{CDCl}_{3}\right)$ 1.66-1.88 (4 H, m, H, H), $2.37(2 \mathrm{H}$, t, $\left.\mathrm{H}_{5}, J_{5-4}=6.5 \mathrm{~Hz}\right), 2.45\left(2 \mathrm{H}, \mathrm{t}, \mathrm{H}_{2}, J_{2-3}=6.5 \mathrm{~Hz}\right), 7.23-7.32\left(3 \mathrm{H}, \mathrm{m}, \mathrm{H}_{3}, 4^{4}\right), 7.34-7.40(2 \mathrm{H}, \mathrm{m}$, $\mathrm{H}_{2}$ ), $\delta_{\mathrm{C}}\left(67.5 \mathrm{MHz}, \mathrm{CDCl}_{3}\right) 16.6 \mathrm{C}_{5}, 18.5 \mathrm{C}_{2}, 24.4,27.3 \mathrm{C}_{3,4}, 81.4 \mathrm{C}_{7}, 88.5 \mathrm{C}_{6}, 119.4 \mathrm{C}_{1}, 123.4$ $\mathrm{C}_{1}, 127.6 \mathrm{C}_{4}, 128.1 \mathrm{C}_{3}, 131.4 \mathrm{C}_{2}, ; \mathrm{m} / \mathrm{z}\left(\mathrm{CI}+, \mathrm{NH}_{3}\right) 201\left(\left[\mathrm{M}+\mathrm{NH}_{4}\right]^{+}, 100\right)$; HRMS (CI+) Found $\left[\mathrm{M}+\mathrm{NH}_{4}\right]^{+} 201.1390 \mathrm{C}_{13} \mathrm{H}_{17} \mathrm{~N}_{2}$ requires 201.1392

5-Ethoxy-2-(6-phenylhex-5-ynyl)-4-triethylsilyloxazole (6c). 7-Phenyl-hept-6-ynenitrile 11 (0.137 g, $0.75 \mathrm{mmol})$, ethyl (triethylsilyl)diazoacetate $(0.114 \mathrm{~g}, 0.5 \mathrm{mmol})$ and $\mathrm{Rh}_{2}$ (octanoate) ${ }_{4}$ ( $4 \mathrm{mg}, 0.005 \mathrm{mmol}$ ) were dissolved in dry benzene $(2 \mathrm{ml})$ and heated to reflux. On consumption of the silyldiazoester by TLC, the reaction was cooled to room temperature and concentrated under reduced pressure. The crude product was purified by flash chromatography (90:10 petroleum ether:diethyl ether) to yield 5-ethoxy-2-(6-phenylhex-5-ynyl)-4-triethylsilyloxazole 6c as a colourless liquid (0.119 g, $62 \%)$. $v_{\max }(\mathrm{KBr} / \mathrm{film}) 2950 \mathrm{~s}, 2874 \mathrm{~s}, 2231 \mathrm{w}(\mathrm{C} \equiv \mathrm{C}), 1605 \mathrm{~s}$, 1574 w, 1461 w, 1244 m, 1018 m, 755 m, 737 m, 724 m, $692 \mathrm{~m} \mathrm{~cm}^{-1}$; $\delta_{\mathrm{H}}\left(300 \mathrm{MHz}, \mathrm{CDCl}_{3}\right)$ $0.72\left(6 \mathrm{H}, \mathrm{q}, J=8 \mathrm{~Hz}, \mathrm{SiCH}_{2} \mathrm{CH}_{3}\right), 0.94\left(9 \mathrm{H}, \mathrm{t}, J=8 \mathrm{~Hz}, \mathrm{SiCH}_{2} \underline{\mathrm{CH}}_{3}\right), 1.32(3 \mathrm{H}, \mathrm{t}, J=7 \mathrm{~Hz}$, $\mathrm{OCH}_{2} \underline{\mathrm{CH}}_{3}$ ), 1.59-1.68 (2 H, m, H $3^{\prime}$ ), 1.81-1.91 (2 H, m, $\left.\mathrm{H}_{2}{ }^{\prime}\right), 2.42$ (2 H, t, $\left.J_{4^{\prime}-3^{\prime}}=7 \mathrm{~Hz}, \mathrm{H}_{4}{ }^{\prime}\right), 2.69$

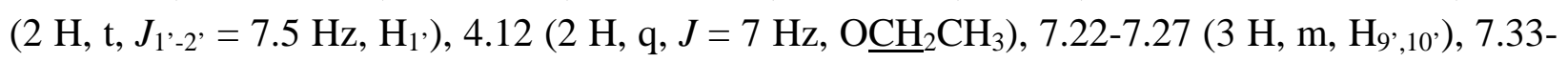
$7.38\left(2 \mathrm{H}, \mathrm{m}, \mathrm{H}_{8}\right.$ ); $\delta_{\mathrm{C}}\left(75 \mathrm{MHz}, \mathrm{CDCl}_{3}\right) 3.1 \mathrm{SiCH}_{2} \mathrm{CH}_{3}, 7.3 \mathrm{SiCH}_{2} \underline{\mathrm{CH}}_{3}, 14.9 \mathrm{OCH}_{2} \underline{\mathrm{CH}_{3}}, 19.0 \mathrm{C}_{4}$, 26.5, 28.1 (2 peaks) $\mathrm{C}_{1}^{\prime}, 2^{\prime}, 3^{\prime}, 69.4 \mathrm{OCH}_{2} \mathrm{CH}_{3}, 80.9 \mathrm{C}_{6}$, $89.7 \mathrm{C}_{5}, 109.5 \mathrm{C}_{4}, 123.9 \mathrm{C}_{7}, 127.5 \mathrm{C}_{10}$, $128.1 \mathrm{C}_{9}, 131.5 \mathrm{C}_{8}, 156.9 \mathrm{C}_{2}, 164.5 \mathrm{C}_{5} ; \mathrm{m} / \mathrm{z}\left(\mathrm{CI}+, \mathrm{NH}_{3}\right) 384$ ([M+H] $\left.]^{+}, 40\right), 354$ (65), 326 (57), 310 (20), 282 ([M-C $\left.{ }_{6} \mathrm{H}_{5} \mathrm{C} \equiv \mathrm{C}^{+}, 19\right), 224$ (14), 115 (56), 103 (33), 87 (40), 75 (42), 49 (100); HRMS (CI+) Found $[\mathrm{M}+\mathrm{H}]^{+} 384.2361 ; \mathrm{C}_{23} \mathrm{H}_{34} \mathrm{NO}_{2} \mathrm{Si}$ requires 384.2359.

2-Ethoxy-3-methoxycarbonyl-6,7-dihydro-5H-benzofuran-4-one (12a). A solution of 5ethoxy-2-(4-oxo-6-methoxycarbonylhex-5-ynyl)-4-triethylsilyloxazole 6a (0.24 g, $0.632 \mathrm{mmol})$ in toluene $(2 \mathrm{ml})$ was heated to $120^{\circ} \mathrm{C}$ and monitored by TLC for complete consumption of the alkyne. The solution was cooled to room temperature, concentrated under reduced pressure and the residual oil purified by flash chromatography (10:90 petroleum ether:diethyl ether) to yield 
2-ethoxy-3-methoxy carbonyl-4-oxo-4,5,6,7-tetrahydrobenzofuran 12a as a white solid (0.093 g, 62 \%). m.p. 67-68 ${ }^{\circ} \mathrm{C}$; $v_{\max }(\mathrm{KBr} / \mathrm{film}) 2973$ w, 2945 w, $2903 \mathrm{w}, 1681 \mathrm{~s}(\mathrm{C}=\mathrm{O}), 1608 \mathrm{~s}(\mathrm{C}=\mathrm{O})$, $1593 \mathrm{~m}, 1451 \mathrm{w}, 1263 \mathrm{~m}, 1224 \mathrm{~m}, 1108 \mathrm{w}, 1085 \mathrm{~m}, 1010 \mathrm{w} \mathrm{cm}^{-1}$; $\delta_{\mathrm{H}}\left(270 \mathrm{MHz}, \mathrm{CDCl}_{3}\right) 1.39$ (3 $\left.\mathrm{H}, \mathrm{t}, J=7 \mathrm{~Hz}, \mathrm{OCH}_{2} \underline{\mathrm{CH}}_{3}\right), 2.09\left(2 \mathrm{H}, \mathrm{p}, J=6.5 \mathrm{~Hz}, \mathrm{H}_{6}\right), 2.45\left(2 \mathrm{H}, \mathrm{t}, J_{7-6}=6.5 \mathrm{~Hz}, \mathrm{H}_{7}\right), 2.73$ (2 $\left.\mathrm{H}, \mathrm{t}, J_{5-6}=6.5 \mathrm{~Hz}, \mathrm{H}_{5}\right), 3.77\left(3 \mathrm{H}, \mathrm{s}, \mathrm{CO}_{2} \mathrm{Me}\right), 4.36\left(2 \mathrm{H}, \mathrm{q}, J=7 \mathrm{~Hz}, \mathrm{OCH}_{2} \mathrm{CH}_{3}\right) ; \delta_{\mathrm{C}}(67.5 \mathrm{MHz}$, $\left.\mathrm{CDCl}_{3}\right) 14.8 \mathrm{OCH}_{2} \underline{\mathrm{CH}_{3}}, 21.9,22.8 \mathrm{C}_{6,7}, 38.3 \mathrm{C}_{5}, 51.5 \mathrm{CO}_{2} \underline{\mathrm{CH}_{3}}, 69.1 \mathrm{OCH}_{2} \mathrm{CH}_{3}, 90.3 \mathrm{C}_{3}, 119.6$ $\mathrm{C}_{3 \mathrm{a}}, 157.8 \mathrm{C}_{7 \mathrm{a}}, 161.7 \mathrm{CO}_{2} \mathrm{CH}_{3}, 162.7 \mathrm{C}_{2}, 191.8 \mathrm{C}=\mathrm{O} ; \mathrm{m} / \mathrm{z}\left(\mathrm{CI}+, \mathrm{NH}_{3}\right) 239\left([\mathrm{M}+\mathrm{H}]^{+}, 100\right)$; HRMS $(\mathrm{CI}+)$ Found $[\mathrm{M}+\mathrm{H}]^{+}$239.0923; $\mathrm{C}_{12} \mathrm{H}_{15} \mathrm{O}_{5}$ requires 239.0920 .

2-Ethoxy-3-trimethylsilyl-6,7-dihydro-5H-benzofuran-4-one (12b). A solution of 2-(4-oxo-6trimethylsilylhex-5-ynyl)-5-ethoxy-4-triethylsilyloxazole $\mathbf{6 b}(0.088 \mathrm{~g}, 0.223 \mathrm{mmol})$ in DCB $(0.5 \mathrm{ml})$ was heated to $180{ }^{\circ} \mathrm{C}$ and monitored by TLC for complete consumption of the alkyne. The solution was cooled to room temperature, concentrated under reduced pressure and the residual oil purified by flash chromatography (70:30 petroleum ether:diethyl ether) to yield 2ethoxy-3-trimethyl silyl-6,7-dihydro-5H-benzofuran-4-one $\mathbf{1 2 b}$ as a colourless liquid (0.032 g, 57 \%). $v_{\max }(\mathrm{KBr} / \mathrm{film}) 2954 \mathrm{~s}, 2898 \mathrm{~m}, 1677 \mathrm{~s}(\mathrm{C}=\mathrm{O}), 1587$ s, $1406 \mathrm{~m}, 1273 \mathrm{~m}, 1242 \mathrm{~s}, 1060 \mathrm{w}$, $1005 \mathrm{~s}, 844 \mathrm{~s}, 762 \mathrm{w} \mathrm{cm}^{-1} ; \delta_{\mathrm{H}}\left(300 \mathrm{MHz}, \mathrm{CDCl}_{3}\right) 0.22\left(9 \mathrm{H}, \mathrm{s}, \mathrm{SiMe}_{3}\right), 1.34(3 \mathrm{H}, \mathrm{t}, J=7 \mathrm{~Hz}$, $\left.\mathrm{OCH}_{2} \underline{\mathrm{CH}}_{3}\right), 2.11\left(2 \mathrm{H}, \mathrm{p}, J=6.5 \mathrm{~Hz}, \mathrm{H}_{6}\right), 2.42\left(2 \mathrm{H}, \mathrm{t}, J_{7-6}=6.5 \mathrm{~Hz}, \mathrm{H}_{7}\right), 2.74\left(2 \mathrm{H}, \mathrm{t}, J_{5-6}=6.5\right.$ $\mathrm{Hz}, \mathrm{H}_{5}$ ), 4.14 (2 H, q, $J=7 \mathrm{~Hz}, \mathrm{OCH}_{2} \mathrm{CH}_{3}$ ); $\delta_{\mathrm{C}}\left(75 \mathrm{MHz}, \mathrm{CDCl}_{3}\right)-0.3 \mathrm{SiMe}_{3}, 14.9 \mathrm{OCH}_{2} \underline{\mathrm{CH}}_{3}$, 22.4, 22.9 $\mathrm{C}_{6,7}, 37.8 \mathrm{C}_{5}, 69.1 \mathrm{OCH}_{2} \mathrm{CH}_{3}, 89.7 \mathrm{C}_{3}, 125.2 \mathrm{C}_{3 \mathrm{a}}, 159.9 \mathrm{C}_{7 \mathrm{a}}, 163.1 \mathrm{C}_{2}, 194.6 \mathrm{C}=\mathrm{O}$; m/z (CI+, $\left.\mathrm{NH}_{3}\right) 253\left([\mathrm{M}+\mathrm{H}]^{+}, 100\right), 237$ (40), 181 ([M-SiMe $\left.\left.3+\mathrm{H}\right]^{+}, 10\right) ; \mathrm{HRMS}(\mathrm{CI}+$ ) Found $[\mathrm{M}+\mathrm{H}]^{+}$253.1259; $\mathrm{C}_{13} \mathrm{H}_{21} \mathrm{O}_{3}$ Si requires 253.1260.

\section{Acknowledgements}

We thank the EPSRC for funding of this project (GR/L60135) and Johnson Matthey plc for the loan of rhodium salts.

\section{References}

1. For reviews of Diels-Alder reactions of oxazoles, see: (a) Boger, D. L. Tetrahedron 1983, 39, 2869. (b) Boger, D. L. Chem. Rev. 1986, 86, 781. (c) Hassner, A.; Fischer, B. Heterocycles 1993, 35, 1441.

2. See, for example: Jacobi, P. A.; Lee, K. J. Am. Chem. Soc. 2000, 122, 4295 and references therein.

3. Huisgen, R.; König, H.; Binsch, G.; Sturm, H. J. Angew. Chem. 1961, 73, 368.

4. Connell, R.; Scavo, F.; Helquist, P.; Akermark, B. Tetrahedron Lett. 1986, 27, 5559.

5. Doyle, K. J.; Moody, C. J. Tetrahedron 1994, 50, 3761.

6. Shankar, B. K. R.; Schechter, H. Tetrahedron Lett. 1982, 23, 2277. 
7. Dewar, M. J. S.; Turchi, I. J. Chem. Rev. 1975, 75, 724.

8. (a) Johnsen, B. A.; Undheim, K. Acta. Chem. Scand., Ser. B 1983, 37, 127. (b) Shimada, S.; Maeda, H. Chem. Pharm. Bull. 1983, 3460.

9. Ducept, P. C.; Marsden, S. P. Synlett 2000, 692.

10. Boyd, G. V. "Oxazoles and their benzo derivatives", In Comprehensive Heterocyclic Chemistry, A. R. Katritzky; C. W. Rees Eds; Pergamon: Oxford; Vol. 5, Ch. 4.18.

11. Nyström, J.-E.; McCanna, T. D.; Helquist, P.; Amoroux, R. Synthesis 1988, 56.

12. Midland, M.; Tramontano, A; Cable, J. R. J.Org.Chem. 1980, 45, 28

13. Crandall, J. K.; Michaely, W. J. J. Org. Chem. 1984, 49, 4244. 